\title{
КВАНТОВО-ЭЛЕКТРОДИНАМИЧЕСКАЯ ТЕОРИЯ ТВЕРДОГО ТЕЛА НА ОСНОВЕ ОРТОГОНАЛИЗИРОВАННЫХ МОДУЛИРОВАННЫХ ПЛОСКИХ ВОЛН
}

\author{
(Представил В. Хижняков)
}

\section{1. Введение}

Построен квантово-электродинамический метод, в котором твердое тело рассматривается как система, состоящая из электронного и ядерного полей, взаимодействующих через вакуум электромагннтного поля.

В основу нового метода положена следующая формула $\left[{ }^{1}\right]$ :

$$
E=\left(\Psi_{0 l}, \hat{H}_{0} \Psi_{0 l}\right)+(i \hbar / 2) \lim _{\tilde{\alpha} \rightarrow 0} \tilde{\alpha}\left(\Psi_{0 l}, \hat{S}_{\tilde{\alpha}} \Psi_{0 l}\right)_{\mathrm{cв}},
$$

где

$$
\hat{S}_{\tilde{\alpha}}=\hat{R}_{\tilde{\alpha}} \sum_{k=0}^{\infty}(-1 / \hbar)^{k} \frac{1}{k !} \int \ldots \underbrace{\infty}_{-\infty} \int d t_{1} \ldots d t_{k} \hat{T} \hat{H}_{i}\left(t_{1}\right) \ldots \hat{H}_{i}\left(t_{k}\right),
$$

дающая связь между собственным значением оператора энергии $\hat{H}$ и матрицей рассеяния $\hat{S}_{\tilde{\alpha}}$, порожденной оператором энергии взаимодействия $\hat{H}_{i}$.

В формуле (1.1) $\hat{H}_{0}$ - оператор энергии свободных полей, $\Psi_{0 l}$ собственный вектор оператора $\hat{H}_{0}, \hat{R}_{\tilde{\alpha}}$ - оператор регуляризации матрицы рассеяния по временам, вставляющий в подынтегральное выражение каждого истинно связного графа Фейнмана регуляризующий множитель $\mathrm{e}^{-\tilde{\alpha}\left|t_{k}\right|}$, где $t_{k}$ - время, по которому в истинно связном графе производится последнее интегрирование, $\tilde{\alpha}>0$. Индекс «св» означает, что из второго члена в правой части формулы (1.1) удалены все несвязные, псевдосвязные и вакуумные графы Фейнмана. Формулу (1.1) можно получить из формул хорошо известной теории возмущений Рэлея-Шредингера и, наоборот, переходя от представления взаимодействия к представлению Шредингера, можно получить из (1.1) ряд теории возмущений Рэлея-Шредингера.

\section{2. Система, состоящая из электронов и ядер $\left[{ }^{2}\right]$}

В квантовой теории поля оператор энергии системы, состоящей из электронов и ядер, представим в следующем виде:

$$
\hat{H}=\hat{H}_{0}+\hat{H}_{i},
$$




$$
\begin{gathered}
\hat{H}_{0}=\hat{H}_{0}^{(e)}+\hat{H}_{0}^{(p)}+\hat{H}_{0}^{(p h)}, \\
\hat{H}_{i}=\hat{H}_{i}^{(e)}+\hat{H}_{i}^{(p)}, \\
\hat{H}_{0}^{(e)}=-i \hbar c \int^{3} x \hat{N} \hat{\psi}\left(\sum_{i=1}^{3} \hat{\gamma}_{i} \frac{\partial}{\partial x_{i}}+x_{0}\right) \hat{\psi}
\end{gathered}
$$

оператор энергии свободного электронного поля $\left[{ }^{2}\right]$,

$$
\hat{H}_{0}^{(p)}=-i \hbar c \int d^{3} x \hat{\bar{N}} \hat{\bar{\Psi}}\left(\sum_{i=1}^{3} \hat{\Gamma}_{i} \frac{\partial}{\partial x_{i}}+X_{0}\right) \hat{\Psi}
$$

- оператор энергии свободного поля узлов решетки,

$$
\hat{H}_{i}^{(e)}=-e_{0} \int d^{3} x \hat{N}\left[\hat{\bar{\psi}} \hat{\gamma_{\mu}} \hat{\psi} \hat{A}_{\mu}\right]
$$

- оператор энергии взаимодействия электронов с электромагнитным полем,

$$
\hat{H}_{i}^{(p)}=e_{0} Z \int d^{3} x \hat{N}\left[\hat{\bar{\Psi}} \hat{\Gamma}_{\mu} \hat{\Psi} \hat{A}_{\mu}\right] \quad=
$$

- оператор энергии взаимодействия ядер с электромагнитным полем, $\hat{H}_{0}^{(p h)}-$ оператор энергии электромагнитного поля.

В формулах $(2.1)-(2.7)$ введены следующие обозначения: $\hat{\psi}-$ оператор электронного поля, $\hat{\Psi}^{\prime}$ - оператор поля узлов решетки (ядер), $\hat{\bar{\Psi}}=\hat{\Psi}+\hat{\Gamma}_{5}, \hat{\Gamma}_{\mu}(\mu=1,2,3,4,5) \quad$ - квадратные матрицы, ранг которых равен $2(2 S+1)$, где $S-$ спин ядра (если $S=1 / 2$, то $\hat{\Gamma}_{\mu}=\hat{\gamma}_{\mu}$ при $\mu=1,2,3,4$ и $\left.\hat{\Gamma}_{5}=\hat{i \hat{\gamma}_{4}}\right) ; \quad \hat{A}_{\mu}$ - оператор электромагнитного поля, $e_{0} Z$ - заряд узла решетки (ядра), $M=\hbar X_{0} / c-$ масса ядра, $m=\hbar x_{0} / c$ - масса электрона, - $e_{0}-$ заряд электрона.

Преобразуем формулу (2.1) к виду

$$
\hat{H}_{1}=\mathscr{H}_{0}+\tilde{H}_{i}+\Delta \hat{H},
$$

где

$$
\tilde{H}_{0}=\mathscr{H}_{0}^{(e)}+\tilde{H}_{0}^{(p)}+\hat{H}_{0}^{(p h)}, \quad \tilde{H}_{i}=\tilde{H}_{i}^{(e)}+\mathscr{H}_{i}^{(p)}
$$

Новые операторы энергии (невозмущенные и взаимодействия) теперь имеют вид:

$$
\begin{aligned}
& \widetilde{H}_{0}^{(e)}=\hat{H}_{0}^{(e)}+\widetilde{H}_{d}^{(e)}+\hat{H}_{e p}^{(e)}, \quad \boldsymbol{H}_{i}^{(e)}=\hat{H}_{i}^{(e)}-\widetilde{H}_{d}^{(e)}, \\
& \mathscr{H}_{0}^{(p)}=\hat{H}_{0}^{(p)}+\mathscr{H}_{d}^{(p)}-\hat{H}_{e p}^{(p)}, \quad \mathscr{H}_{i}^{(p)}=\hat{H}_{i}^{(p)}-\mathscr{H}_{d}^{(p)}, \\
& \Delta \hat{H}=\hat{H}_{e p}^{(p)}-\hat{H}_{e p}^{(e)} .
\end{aligned}
$$


В формулы (2.10)-(2.12) входят следующие операторные члены: a) оператор энергии взаимодействия между электронами системы

$$
\widetilde{H}_{d}^{(e)}=\int d^{3} x \hat{N} \hat{\psi}_{d}^{+} \tilde{U}_{d}^{(e)}(\boldsymbol{x}) \hat{\psi},
$$

учитывающий их кулоновскую, обменную и корреляционную энергии $(\boldsymbol{x}$ - радиус-вектор в 3-мерном пространстве),

б) оператор энергии взаимодействия между свободными ядрами

$$
\tilde{H}_{d}^{(p)}=\int d^{3} x \hat{N} \hat{\Psi}^{+}+\tilde{U}_{d}^{(p)}(x) \hat{\Psi}
$$

в) оператор энергии взаимодействия электронов с зафиксированными ядрами (узлами решетки) в $\hat{\psi}$-представлении:

$$
\hat{H}_{e p}^{(e)_{i}}=-e_{0}^{2} Z \int d^{3} x \hat{N} \hat{\psi}^{+} L_{r}(x) \hat{\psi},
$$

где

$$
L_{r}(x)=\sum_{l}|x-l|^{-1}-\sum_{l \neq 0}|l|^{-1}
$$

$\boldsymbol{l}$ - радиус-вектор положения ядра (узла решетки),

г) оператор энергии взаимодействия электронов с зафиксированными

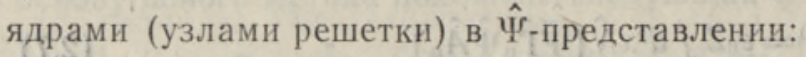

$$
\hat{H}_{e p}^{(p)}=-e_{0}^{2} Z^{2} \int d^{3} x_{1 \varrho}\left(x_{1}\right) L_{r}\left(x_{1}\right) \int d^{3} x_{2} \hat{N}(\hat{\Psi}+\hat{\Psi})_{2},
$$

где $\varrho(\boldsymbol{x})-$ плотность электронов.

Операторы $\tilde{H}_{0}^{(e)}$ и $\tilde{H}_{0}{ }^{(p)}-$ эффективные операторы: энергии соответственно электронного поля и поля ядер.

Операторы $\tilde{H}_{i}^{(e)}$ и $\tilde{H}_{i}{ }^{(p)}$ - эффективные операторы энергии взаимодействия соответственно электронного поля и поля ядер с электромагнитным полем.

В дальнейшем оператором $\Delta \hat{H}$ будем пренебрегать ввиду его малости, что следует из формул (2.15) и (2.16). В задачах о твердом теле функцин $\widetilde{D}_{d}^{(e)}(\boldsymbol{x})$ и $\widetilde{\delta}_{d}^{(p)}(\boldsymbol{x})$ в формулах $(2.13)$ и $(2.14)$ - периодические функции с периодом решетки $a$.

Пусть $\Psi_{0 l}$ - собственный вектор оператора энергии $\mathscr{H}_{0}$. Так как операторы энергии $\tilde{H}_{0}^{(e)}$ и $\widetilde{H}_{0}^{(p)}$ действуют на разные «координаты», то можно произвести разделение переменных и представить $\Psi_{0 l}$ в виде

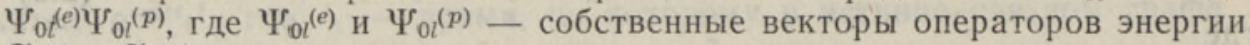
$\tilde{H}_{0}{ }^{(e)}$ и $\tilde{H}_{0}{ }^{(p)}$ соответственно.

Учитывая сказанное и используя формулу (1.1), для собственного значения оператора энергии $\tilde{H}_{0}+\tilde{H}_{i}$ находим следующую формулу:

$$
\begin{gathered}
E_{l}=\left(\Psi_{0 l}^{(e)}, \tilde{H}_{0}^{(e)} \Psi_{0 l}^{(e)}\right)+\left(\Psi_{0 l}^{(p)}, \tilde{H}_{0}^{(p)} \Psi_{0 l}^{(p)}\right)+ \\
+(i \hbar / 2) \lim _{\tilde{\alpha} \rightarrow 0} \tilde{\alpha}\left(\Psi_{0 l}^{(e)}, \hat{S}_{\tilde{\alpha} e} \Psi_{0 l}^{(e)}\right)_{\mathrm{cB}}+(i \hbar / 2) \underset{\tilde{\alpha} \rightarrow 0}{\lim _{\tilde{\omega}} \tilde{\alpha}\left(\Psi_{0 l}^{(p)}, \hat{S}_{\tilde{\alpha} p} \Psi_{0 l}^{(p)}\right)_{\mathrm{cB}}+} \\
+(i \hbar / 2) \lim _{\tilde{\alpha} \rightarrow 0} \tilde{\alpha}\left(\Psi_{0 l}, \hat{S}_{\tilde{\alpha}}^{\text {cross }} \Psi_{0 l}\right)_{\mathrm{cB}},
\end{gathered}
$$

где $\hat{S}_{\tilde{\alpha} e}$ и $\hat{S}_{\tilde{\alpha} p} \quad$ - матрицы рассеяния,// порожденные операторами 
энергии взаимодействия $\tilde{H}_{i}^{(e)}$ и $\tilde{H}_{i}{ }^{(p)}$ соответственно. Последний член в правой части формулы (2.17) - сумма всех диаграмм Фейнмана, на которых фотонные связки соединяют электронные линии с ядерными.

Вид оператора $\hat{H}_{e p}{ }^{(p)}$ показывает, что вектор состояния $\Psi_{0 l}{ }^{(p)}$ является собственным вектором как оператора $\tilde{H}_{0}(p)$, так и оператора

$$
\check{H}_{0}^{(p)}=\hat{H}_{0}^{(p)}+\mathscr{H}_{d}^{(p)} \text {. }
$$

Учитывая это, из (2.17) получаем окончательное выражение для собственного значения оператора энергии $(2.8)$ :

$$
\begin{aligned}
& E_{l}=\left(\Psi_{0 l}^{(e)}, \tilde{H}_{0}^{(e)} \Psi_{0 l}^{(e)}\right)+(i \hbar / 2) \lim _{\tilde{\alpha} \rightarrow 0} \tilde{\alpha}\left(\Psi_{0 l}^{(e)}, \hat{S}_{\tilde{\alpha} e} \Psi_{0 l}^{(e)}\right)_{\mathrm{cB}}+ \\
& +\left(\Psi_{0 l}^{(p)}, \check{H}_{0}^{(p)} \Psi_{0 l}^{(p)}\right)+(i \hbar / 2) \underset{\tilde{\alpha}_{\leftrightarrow \rightarrow 0}}{\lim } \tilde{\alpha}\left(\Psi_{0 l}^{(p)}, \hat{S}_{\tilde{\alpha} p} \Psi_{0 l}^{(p)}\right)_{\mathrm{cB}}+ \\
& +\left[(i \hbar / 2) \lim _{\tilde{\alpha} \rightarrow 0} \tilde{\alpha}\left(\Psi_{0 l}, \hat{S}_{\tilde{\alpha}}^{\text {cross }} \Psi_{0 l}\right)_{\mathrm{cB}}-\left(\Psi_{0 l}^{(p)}, \hat{H}_{e p}^{(p)} \Psi_{0 l}^{(p)}\right)\right] .
\end{aligned}
$$

В (2.19) первые два члена вносят вклад в энергию электронов, третий и четвертый члены - в энергию системы ядер, а последний член является неадиабатической энергией, характеризующей взаимодействие электронов с колебаниями системы ядер. Используя формулу (2.19), мы фактически выходим за пределы адиабатического приближения, а пренебрегая в ней последним членом, переходим к адиабатическому приближению.

Әнергия системы электронов и ядер, согласно формуле (2.19), во втором приближении теории возмущений равна

$$
E_{l}=E_{0}^{(e)}+E_{1}^{(e)}+E_{2}^{(e)}+E_{0}^{(p)}+E_{1}^{(p)}+E_{2}^{(p)}+E_{\text {на, }}
$$

где

$$
\begin{aligned}
& E_{0}^{(e)}=\left(\Psi_{0 l}^{(e)}, \mathscr{H}_{0}^{(e)} \Psi_{0 l}^{(e)}\right), \quad E_{0}^{(p)}=\left(\Psi_{0 l}^{(p)}, \check{H}_{0}^{(p)} \Psi_{0 l}^{(p)}\right) \\
& E_{j}^{(e)}=(i \hbar / 2) \lim _{\tilde{\alpha} \rightarrow 0} \tilde{\alpha}\left(\Psi_{0 l}^{(e)}, \hat{S}_{e, j \tilde{\alpha}} \Psi_{0 l}^{(e)}\right)_{\text {св }} \quad(j=1,2), \\
& E_{j}^{(p)}=(i \hbar / 2) \lim _{\tilde{\alpha} \rightarrow 0} \tilde{\alpha}\left(\Psi_{0 l}^{(p)}, \hat{S}_{p, j \tilde{\alpha}} \Psi_{0 l}^{(p)}\right)_{\mathrm{cв}} \quad(j=1,2), \\
& E_{\text {на }}=(i \hbar / 2) \lim _{\alpha \rightarrow 0} \tilde{\alpha}\left(\Psi_{0 l}, \hat{S}_{2 \tilde{\alpha}}^{c r o s s} \Psi_{0 l}\right)_{\mathrm{cв}}-\left(\Psi_{0 l}^{(p)}, \hat{H}_{e p}^{(p)} \Psi_{0 l}^{(p)}\right),
\end{aligned}
$$

$\hat{S}_{e+\tilde{\alpha}}, \hat{S}_{e 2 \tilde{\alpha}}$ - второй и третий члены матрицы рассеяния, порожденной оператором энергии взаимодействия $\mathscr{H}_{i}^{(e)} ; \hat{S}_{p 1 \tilde{\alpha}}$ и $\hat{S}_{p 2 \tilde{\alpha}}-$ те же члены, порожденные оператором энергии взаимодействия $\tilde{H}_{i}(p)$.

В операторах энергии $\tilde{H}_{0}^{(e)}$ и $\tilde{H}_{i}^{(e)}$ оператор электронного поля $\hat{\psi}$, следует разлагать в ряд по полной системе функций, являющихся решениями одноэлектронного уравнения с потенциальной іэнергией $U_{r}^{(e)}(\boldsymbol{x})=\widetilde{U}_{r}^{(e)}(\boldsymbol{x})-e_{0}^{2} Z_{r} L_{r}(\boldsymbol{x})$. Аналогично, в операторах энергии 
$\check{H}_{0}{ }^{(p)}$ и $\hat{H}_{i}{ }^{(p)}$ оператор поля $\hat{\Psi}$ следует разлагать в ряд по полной системе функций $\left\{\Psi_{\beta}\right\}$, являющихся решениями одноядерного (одноузельного) уравнения с потенциальной энергией $\widetilde{\sigma}_{r}^{(p)}(\boldsymbol{x})$.

\section{3. Вычисление выражения $\boldsymbol{E}_{0}^{(e)}$}

Для вычисления выражения $E_{0}{ }^{(e)}$, входящего в $(2.20)$ и имеющего вид (2.21), будем разлагать оператор электронного поля $\hat{\psi}$ в ряд по системе ортогонализированных модулированных плоских волн (ОМПВ) [ $\left.{ }^{3}\right]$.

ОМПВ в самом общем виде можно записать так:

$$
K_{k s}=\sum_{j=1}^{n} C_{j} K_{k s}^{(j)}
$$

где

$$
K_{k s}^{(j)}=N_{y}^{-1 / 2} \sum_{l} e^{i k l \beta /} \varphi^{(j)}(x-l) e i k x \tilde{\beta_{t}} C_{s} \quad(j=1, \ldots, n)
$$

$\left\{C_{j}\right\}$ - искомые коэффициенты, $\beta_{j}$ и $\tilde{\beta}_{j}-$ некоторые параметры $\left(\beta_{j}+\tilde{\beta}_{j}=1\right),\left\{\varphi^{(j)}(\boldsymbol{x})\right\}$ - атомные волновые функции $s-, p-, d-$ и $f$-состояний, $N_{y}$ - число узлов решетки, $C_{s}-$ спиновая функция, $\hbar k-$ квазиимпульс электрона.

В общем случае $C_{j}$ и $\beta_{j}-$ функции волнового числа $k$.

Для остовных (внутренних) одноэлектронных состояний можно положить $\beta_{j}=1$ и $\tilde{\beta}_{j}=0$ (при этом все $C_{j}$ равны нулю, кроме одного). В этом случае функция (3.1) превращается в блоховскую функцию типа ЛКАО.

В нерелятивистском приближении функция (3.1) должна быть собственной функцией одноэлектронного уравнения Шредингера с эффективной потенциальной энергией электрона

$$
\underset{r}{U(e)}=(x)=-e_{0}^{2} Z L_{r}(x)+\widetilde{U}_{r}^{(e)}(x),
$$

где $-e_{0}^{2} Z L_{r}(\boldsymbol{x})$ и $\widetilde{\boldsymbol{U}}_{r}^{(e)}(\boldsymbol{x})$ - энергии взаимодействия электрона с узлами решетки и с остальными электронами в твердом теле соответственно.

Перейдем к приближению, в котором

$$
\widetilde{U}_{r}^{(e)}(x) \approx e_{0}^{2} \delta_{r} L_{r}(x),
$$

где $\delta_{r}-$ некоторый параметр. Согласно (3.4), формула (3.3) принимает вид

$$
U_{r}^{(e)}(x)=-e_{0}^{2} Z_{r}^{*} L_{r}(x),
$$

где $Z_{r}{ }^{*}=Z-\delta_{r}, e_{0} Z_{r}{ }^{*}-$ эффективный заряд ядра (узла решетки).

Одноэлектронные состояния в твердом теле будем делить на внутренние (остовные) и внешние (коллективизированные). Внутренние состояния будем также делить на группы $(r=1, \ldots, \tilde{n}-1)$. Потребуем, чтобы выполнялись равенства ортогонализации:

$$
\left(K_{k^{\prime} s}, B_{k s}^{(r)}\right)=0 \quad\left(r=1, \ldots, \tilde{n}-1,\{k\},\left\{k^{\prime}\right\}\right),
$$

где $\left\{B_{k s}^{(r)}\right\}$ и $\left\{K_{k^{\prime} s}\right\}$ - волновые функции внутренних и внешних одноэлектронных состояний соответственно.

Так как атомные волновые функции внутренних электронов, взятые 
для соседних узлов решетки, перекрываются очень слабо, то, согласно (3.5), энергия остовного электрона $r$-й группы в твердом теле равна

$$
E^{\left(B_{r}\right)}=-e_{0}^{2} Z_{r}^{* 2} / 2 a_{0} n^{2},
$$

где $n$ - главное квантовое число, $a_{0}$ - радиус первой орбиты Бора. Әнергия внешнего электрона в твердом теле равна минимуму функционала

$$
E_{k s}^{(K)}=\left(K_{k s}, K_{k s}\right)^{-1}\left(K_{k s},\left[-\frac{\hbar^{2}}{2 m} \nabla^{2}+U_{r}^{(e)}(x)\right] K_{k s}\right)
$$

при выполнении условий (3.6).

Коэффициенты $C_{j}$ и параметры $\beta_{j}$, входящие в $K_{k s}(x)$, необходимо подобрать так, чтобы при соблюдении (3.6) выражение (3.8) достигло минимума. При этом $\underset{k s}{E_{(K)}}$ превращается в функцию только вариационного параметра $\delta_{K}$ и постоянных решетки $\left\{a_{i}\right\}$.

(4 Согласно $(2.21)$, (3.7) и (3.8), энергия системы электронов в нулевом приближении теории возмущений выражается формулой

$$
\begin{gathered}
E_{l}^{(e)}=\left(\Psi_{0 l}^{(e)}, \tilde{H}_{0}^{(e)} \Psi_{0 l}^{(e)}\right)= \\
=\sum_{r} \sum_{k s}^{\left(B_{r}\right)} E_{k s}^{\left(B_{r}\right)} N_{k s}^{\left(B_{r}\right)}+\sum_{j} \sum_{k s}^{(K)} E_{k s, j}^{(K)} N_{k s, j}^{(K)},
\end{gathered}
$$

где $N_{k s}^{\left(B_{r}\right)}$ и $N_{k s, j}^{(K)} \quad-$ числа заполнения остовных и коллективизированных одноэлектронных состояний. Индексы $B_{r}$ и $K$ над суммой означают, что суммирование по $k$ в первом и втором членах правой части (3.9) производится по $B$ - и $K$-состояниям соответственно.

Для основного состояния электронов в твердом теле формула (3.9) принимает вид

$$
E_{0}^{(e)}=\sum_{r} \sum_{k s}^{(B)} E_{k s}^{\left(B_{r}\right)}+\sum_{k s}^{(K)} E_{k s}^{(K)},
$$

где звездочка у суммы означает, что суммирование по $\boldsymbol{k}$ производится по области, ограниченной замкнутой поверхностью (в первой сумме эта поверхность ограничивает несколько зон Бриллюэна, а во второй является поверхностью Ферми).

\section{4. Вычисление выражения $\boldsymbol{E}_{0}(p)$}

Для вычисления выражения $E_{0}(p)$, входящего в $(2.20)$ и имеющего вид $(2.21)$, построим функцию $\widetilde{V}_{d}^{(p)}(\boldsymbol{x})$. Она должна состоять из двух частей:

$$
\tilde{\nabla}_{d}^{(p)}\left(x^{\prime}\right)=\tilde{U}_{d, p}^{(p)}\left(x^{\prime}\right)+\tilde{U}_{d, e}^{(p)}\left(x^{\prime}\right),
$$

где $\tilde{U}_{d, e}^{(p)}\left(x^{\prime}\right)$ и $\tilde{U}_{d, p}^{(p)}\left(x^{\prime}\right)$ - вклады в $\tilde{U}_{d}^{(p)}\left(x^{\prime}\right)$ электронов и узлов решетки соответственно, $\boldsymbol{x}^{\prime}$ - радиус-вектор узла $A$ (т. е. вектор смещения узла $A$ при колебаниях).

Можно показать, что для идеальной кубической решетки

$$
\tilde{D}_{d, p}^{(p)}\left(x^{\prime}\right) \approx 0 .
$$

Отсюда следует, что 


$$
\begin{aligned}
& \tilde{U}_{d}^{(p)}\left(x^{\prime}\right) \approx \tilde{U}_{d, e}^{(p)}\left(x^{\prime}\right)=\sum_{r} \sum_{k s}^{\{B r\}}\left\{\left(\widetilde{B}_{k s}^{(r)}, \tilde{J} \widetilde{B}_{k s}^{(r)}\right)-\left(B_{k s}^{(r)}, \quad \hat{\mathscr{H}}_{0} B_{k s}^{(r)}\right)\right\}+
\end{aligned}
$$

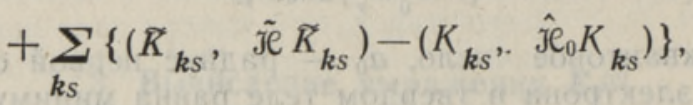

где $\left\{B_{k s}^{(r)}\right\}$ и $\left\{K_{k s}\right\}$ - блоховские функции остовных и коллективизированных одноэлектронных состояний; $\left\{\widetilde{B}_{k s}^{(r)}\right\}$ и $\left\{\widetilde{K}_{k s}\right\}-$ волновыс функции остовных и коллективизированных состояний электрона, движущегося в поле решетки, в которой один узел отклонен от равновесного положения на вектор $\boldsymbol{x}^{\prime} ; \hat{\jmath}_{0}-$ одноэлектронный оператор энергии с потенциальной энергией $(3.5) ; \tilde{\mathcal{H}}-$ то же с потенциальной энергией

$$
\check{U}_{r}^{(e)}(x)=-e_{0}^{2} Z_{r}^{*}\left\{\sum_{l \neq 0}|x-l|^{-1}+\left|x-x^{\prime}\right|^{-1}-\sum_{l \neq 0}|l|^{-1}\right\} .
$$

Выполняя элементарные и громоздкие вычисления, из (4.3) получаем

$$
\widetilde{U}_{d}^{(p)}\left(x^{\prime}\right) \approx(4 \pi / 3) e_{0}^{2} Z_{K}^{*} \sum_{l \neq 0} \Phi_{K}^{2}(l) x^{\prime 2},
$$

где $\Phi_{K}(l)=\sum_{j} C_{j} \varphi_{j}(l) ;\left\{\varphi_{j}(x)\right\}$ - атомные волновые функции для коллективизированных состояний.

Согласно (4.5), узел решетки совершает гармонические колебания под действием упругой силы, пропорциональной вектору отклонения $\boldsymbol{x}^{\prime}$. По общей теории гармонического осциллятора его энергия, т. е. энергия узла решетки в наинизшем состоянии, равна

$$
E_{0}=3 \hbar \tilde{\Omega} / 2 \text {, }
$$

где

$$
\tilde{\Omega}=\sqrt{(8 \pi / 3 M) e_{0}^{2} Z_{K}^{*} \sum_{l \neq 0} \Phi_{K}^{2}(l)}
$$

- угловая частота колебаний, $M$ - масса узла решетки.

Волновая функция узла решетки, согласно (4.5), имеет вид

$$
\Psi\left(x^{\prime}-l\right)=(2 \Omega / \pi)^{3 / 4} e-\Omega\left|x^{\prime}-l\right|^{2},
$$

где $\Omega=\tilde{\Omega} M / 2 \hbar$.

Согласно $(4.6)$, энергия решетки в наинизшем состоянии выражается формулой

$$
E_{0}^{(p)}=3 \hbar \tilde{\Omega} N_{y} / 2
$$

5. Вычисление выражений $\boldsymbol{E}_{1}^{(e)}, \boldsymbol{E}_{1}{ }^{(p)}, \boldsymbol{E}_{2}^{(e)}, \boldsymbol{E}_{2}{ }^{(p)}$ и $\boldsymbol{E}_{\text {на }}$

Согласно $(2.22)$, выражения $E_{1}^{(e)}$ и $E_{2}^{(e)}$, входящие в $(2.20)$, имеют вид

$$
E_{1}^{(e)}=-\sum_{r} \sum_{k s}^{\left(B_{r}\right)}\left(B_{k s}^{(r)}, \sigma_{B}^{(e)} B_{k s}^{(r)}\right)-\sum_{k s}^{(K)}\left(K_{k s}, \tilde{U}_{K}^{(e)} K_{k s}\right),
$$




$$
E_{2}^{(e)} \approx X_{1}+X_{2}
$$

где

$$
X_{1}=-\left(i e_{0}^{2} / 4 \hbar c^{2}\right) \times
$$

$\times \lim _{\tilde{\alpha} \rightarrow 0} \tilde{\alpha}\left(\Psi_{0 l}, \int d^{4} x_{1} \int d^{4} x_{2} \hat{N}\left(\hat{\bar{\psi}} \hat{\psi_{\mu}} \hat{\psi} \hat{A}_{\mu}\right)_{1}^{\tilde{\alpha}}\left(\hat{\bar{\psi}} \hat{\psi} \mu \hat{\psi} \hat{A}_{\mu}\right)_{2} \Psi_{0 l}\right)_{\text {св }}$

- энергия кулоновского и обменного взаимодействия электронов,

$$
X_{2}=\left(i / 2 \hbar c^{2}\right) \times
$$

$\left.\times \lim _{\tilde{\alpha} \rightarrow 0} \tilde{\alpha}\left(\Psi_{0 l}, \int d^{4} x_{1} \int d^{4} x_{2} \hat{N} \hat{\bar{\psi}} \hat{\beta} \bar{U}_{d}^{(e)} \hat{\bar{\psi}}\right)_{1}^{\tilde{\alpha}}\left(\hat{\bar{\psi}} \hat{\beta} \tilde{U}_{d}^{(e)} \hat{\psi}\right)_{2} \Psi_{0 l}\right)_{\text {св }}$

- корреляционная энергия электронов, $\quad(\hat{\bar{\psi}} \hat{O} \hat{\psi})_{1}^{\tilde{\alpha}}=(\hat{\bar{\psi}} \hat{O} \hat{\psi})_{1} \exp \left(-\tilde{\alpha}\left|t_{1}\right|\right)$. вид

Согласно $(2.23)$, выражения $E_{1}{ }^{(p)}$ и $E_{2}{ }^{(p)}$, входящие в $(2.20)$, имеют

$$
\begin{aligned}
& E_{1}^{(p)}=-\left(\Psi_{m}, \sigma_{d}^{(p)} \Psi_{m}\right) N_{y}=-3 \hbar \tilde{\Omega} N_{y} / 4, \\
& E_{2}^{(p)} \approx X_{3}+X_{4},
\end{aligned}
$$

где

$$
X_{3}=-\left(i e_{0}^{2} Z^{2} / 4 \hbar c^{2}\right) X
$$

$$
\times \lim _{\tilde{\alpha} \rightarrow 0} \tilde{\alpha}\left(\Psi_{0 l}, \int d^{4} x_{1} \int d^{4} x_{2} \hat{N}\left(\hat{\bar{\Psi}} \hat{\Gamma}_{\mu} \hat{\Psi} \hat{A}_{\mu}\right)_{1}^{\tilde{\alpha}}\left(\hat{\bar{\Psi}} \hat{\Gamma}_{\mu} \hat{\Psi} \hat{A}_{\mu}\right)_{2} \Psi_{0 l}\right)_{\mathrm{cB}},
$$

- энергия кулоновского и обменного взаимодействия узлов,

$$
X_{4}=\frac{\left(i / 2 \hbar c^{2}\right) \times}{1.1}
$$

$\times \lim _{\tilde{\alpha} \rightarrow 0} \tilde{\alpha}\left(\Psi_{0 l}, \int d^{4} x_{1} \int d^{4} x_{2} \hat{N}\left(\hat{\bar{\Psi}} \sigma_{d}^{(p)} \hat{\Gamma}_{4} \hat{\Psi}\right) \tilde{\alpha}{ }_{1}\left(\hat{\bar{\Psi}} \tilde{U}_{d}^{(p)} \hat{\Gamma}_{4} \hat{\Psi}\right)_{2} \Psi_{0 l}\right)_{\mathrm{CB}}=3 \hbar \tilde{\Omega} N_{y} / 4$

- корреляционная энергия узлов.

Для $X_{3}$ расчет дал формулу

$$
X_{3} \approx\left(Z^{2} / 2\right) e_{0}^{2} N_{y} \sum_{l \neq 0}|l|^{-1}
$$

Переходя к рассмотрению (2.24), легко показать, что во втором приближении теории возмущений по теореме Вика получаем

$$
\text { (iћ/2) } \lim _{\tilde{\alpha} \rightarrow 0} \tilde{\alpha}\left(\Psi_{0 l}, \hat{S}_{2 \tilde{\alpha}}^{\text {cross }} \Psi_{0 l}\right)_{\text {св }} \approx X_{5}
$$




$$
X_{5}=\left(i e_{0}^{2} Z / 2 \hbar c^{2}\right) X
$$

$\times \lim _{\tilde{\alpha} \rightarrow 0} \tilde{\alpha}\left(\Psi_{0 l} \int d^{4} x_{1} \int d^{4} x_{2} \hat{N} \hat{(\bar{\psi} \hat{\psi}} \hat{\psi} \hat{\psi} \hat{A}_{\mu} \tilde{\alpha}_{1}^{\tilde{\alpha}}\left(\hat{\bar{\Psi}} \hat{\Gamma}_{\mu} \hat{\Psi} \hat{A}_{\mu}\right)_{2} \Psi_{0 l}\right)_{\text {св }}$.

Вычитая из $X_{5}$ выражение, расходящееся при $V \rightarrow \infty$, получаем $X_{5, \text { reg. }}$.

Согласно (5.11) имеем:

$$
E_{\text {Ha }}=X_{5}-\lim _{\Omega \rightarrow \infty} X_{5, \text { reg }} .
$$

Учитывая формулы (3.10), (4.9), (5.1)-(5.9) и (5.11), (5.12), находим выражение для энергии твердого тела в основном состоянии (во втором приближении теории возмущений):

$$
E_{0}=\sum_{r} \sum_{k s}^{(B)} E_{k s}^{\left(B_{r}\right)}+\sum_{k s}^{(K)} E_{k s}^{(K)}+E_{0}^{(p)}+\varepsilon_{0},
$$

где

$$
\mathcal{E}_{0}=E_{1}^{(e)}+X_{1}+X_{2}+E_{1}^{(p)}+X_{3}+X_{4}+E_{\text {на }} .
$$

Легко доказать, что

$$
\begin{gathered}
E_{1}^{(p)}+X_{4}=0, \\
X_{1, \infty}+X_{3}+E_{\text {па, } \infty}=0,
\end{gathered}
$$

где $X_{1, \infty}$ и $E_{\text {на, }}$ - части выраженнй $X_{1}$ и $E_{\text {на }}$, расходящиеся при $V \rightarrow \infty$. Кроме того, величины $E_{1}^{(e)}$ и $\left(X_{1, \text { reg }}+X_{2}\right)$ можно представить так:

$$
E_{1}^{(e)}=\sum_{r=1}^{\tilde{n}} E_{1, r}^{(e)}, \quad X_{1, \text { reg }}+X_{2}=\sum_{r=1}^{\tilde{n}} E_{2, r}^{(e)},
$$

где $X_{1, \text { reg }}=X_{1}-X_{1, \infty}, E_{j, r^{(e)}}$ - вклад в энергию $r$-й группы электронов в $j$-м приближении теории возмущений $(j=1,2)$.

Используя формулы (5.15) - (5.17), преобразуем (5.14) к виду

$$
\varepsilon_{0}=\sum_{r=1}^{\tilde{n}}\left(E_{1, r}^{(e)}+E_{2, r}^{(e)}\right)+E_{\text {на, }, \text { eg }, .}
$$

где $E_{\text {на, геg }}=E_{\text {на }}-E_{\text {на, }}$.

Функция $E_{0}$ зависит от параметров $\left\{\delta_{r}\right\}$ и от постоянных решетки $\left\{a_{i}\right\}$. Для того чтобы каждая функция $\tilde{D}_{r}^{(e)}$ имела смысл энергии электрона $r$-й группы в поле остальных электронов твердого тела, выбираем параметры $\left\{\delta_{r}\right\}$ так, чтобы выполнялись дополнительные условия:

$$
E_{1, r}^{(e)}+E_{2, r}^{(e)}=0, \quad r=1, \ldots, \tilde{n} .
$$

Учитывая (5.18) и (5.19), из (5.13) получаем

$$
E_{0}=\sum_{r=1}^{n-1} \sum_{k s}^{(B)} E_{k s}^{\left(B_{r}\right)}+\sum_{k s}^{(K)} E_{k s}^{(K)}+E_{0}^{(p)}+E_{\text {на, reg. }}
$$




\section{6. Заключениё}

Решая систему уравнений $(5.19)$, находим параметры $\left\{\delta_{r}\right\}$, которые подставляем в $(5.20)$. В итоге $E_{0}$ становится функцией только $\left\{a_{i}\right\}$. При равновесных значениях $\left\{a_{i}\right\} E_{0}$ достигает минимума, который является энергией твердого тела в основном состоянии.

Предложенный здесь метод обладает достоинствами, которые позволяют:

1. Определять энергетический электронный спектр твердого тела в замкнутой аналитической форме (3.8), удобной для выполнения расчетов физических свойств твердых тел.

2. Находить параметры, входящие в (5.20), вариационным методом без привлечения подгоночных коэффициентов, определяемых из эксперимента.

3. Определять обменную энергию электронной системы без привлечения слейтеровского приближения.

4. Сократить объем вычислительных работ.

Теория, рассмотренная в данной работе, была использована для расчета энергетического электронного спектра твердого лития с объемноцентрированной кубической структурой (ОЦК) и бериллия с гексагональной плотноупакованной структурой. Найдено, что энергия основного состояния ОЦК лития равна

$$
E_{0}=-7,507\left(e_{0}^{2} N_{y} / a_{0}\right)
$$

при постоянной решетки $a=3,539 \AA$.

Эксперимент для этих величин дает следующие значения:

$$
E_{0, \exp }=-7,5\left(e_{0}^{2} N_{y} / a_{0}\right), \quad a=3,509 \AA .
$$

Заметим, что в качестве ортонормированной системы одноэлектронных функций (вместо ЛКАО + ОМПВ) можно взять множество $G$, в состав которого входят плоские волны $|\boldsymbol{k}\rangle$ и одноэлектронные функции

$$
\widetilde{B}_{k}^{(r)}=B_{k}^{(r)}-\sum_{k^{\prime}}\left\langle k^{\prime} \mid B_{k}^{(r)}\right\rangle\left|k^{\prime}\right\rangle-\sum_{l=r+1}^{\tilde{n}=1} \sum_{k^{\prime}}\left\langle\widetilde{B}_{k^{\prime}}^{(l)} \mid B_{k}^{(r)}\right\rangle \widetilde{B}_{k^{\prime}}^{(l)},
$$

описывающие в нулевом приближении теории возмущений соответственно внешние и остовные электроны. Здесь $\left\{B_{k}^{(r)}\right\}-$ система волновых функций Блоха остовных электронов $r$-й группы типа ЛҚАО, зависящих от некоторых параметров; $\tilde{n}-1$ - число подмножеств остовных электронов.

\section{Л ИТ Е РАТ У РА}

1. К ушн и рен ко А. Н., Препринт ИПМ АН УССР, № 1, Киев, 1976; Изв. ВУЗов, Физнка, № 6, 11-15 (1980).

2. К ушн н рен ко А. Н., Препринт ИПМ АН УССР, № 1, Киев, 1979.

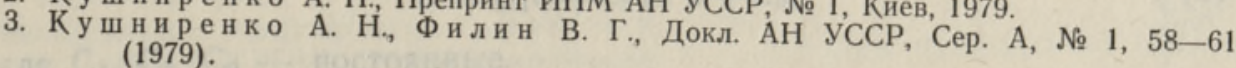

Институт проблем материаловедения Академии наук Украинской ССР
Поступила в редакцию 4/I 1981 


\section{ORTOGONAALMODULEERITUD TASANDLAINEL POHINEV TAHKE KEHA KVANTELEKTRODUNAAMILINE TEOORIA}

Artiklis esitatud meetodil on võimalik kindlaks määrata tahke keha energeetiline elektronspekter kinnisel analüütilisel kujul (3.8), mis sobib tahke keha füüsikaliste omaduste arvutamiseks, leida variatsioonmeetodil, eksperimendis saadud sobituskoefitsienti kasutamata, valemis (5.20) sisalduvad parameetrid ja kindlaks määrata elektronsüsteemi vahetusenergia Slateri lähendust kasutamata, ühtlasi vähendab see meetod arvutuste mahtu.

\section{A. KUSHNIRENKO}

\section{QUANTUM ELECTRODYNAMICAL THEORY OF SOLID ON THE BASIS OF ORTHOGONAL MODULATED PLANE WAVES}

An attempt is made to build a quantum electrodynamical theory of the solid. In this theory the solid is considered as the system of the electron and «lattice» (nuclear) fields interacting with an electromagnetic field according to Yukawa law. The theory is based on the relation proposed by the author, which gives the relation between energy level of the system under consideration and scattering matrix.

In this work all one-electron states are divided into internal (basic) and external (collective) ones. External states are described by orthogonal modulated plane waves, whereas internal states are described by a linear combination of the atomic functions (LCAF). To determine parameters entering into one-electron wave functions a variation method is used.

The advantages of the proposed method consist of possibilities

1) to define the energy electron spectrum of the solid in a closed analytical form, which would be useful for calculating physical properties of the solid;

2) to find parameters entering the expression for energy of the electron system by the variation method without using the phenomenological coefficient defined from experiments;

3) to define the exchange energy of the electron system without using the Slater approximation;

4) to decrease the volume of the computational work.

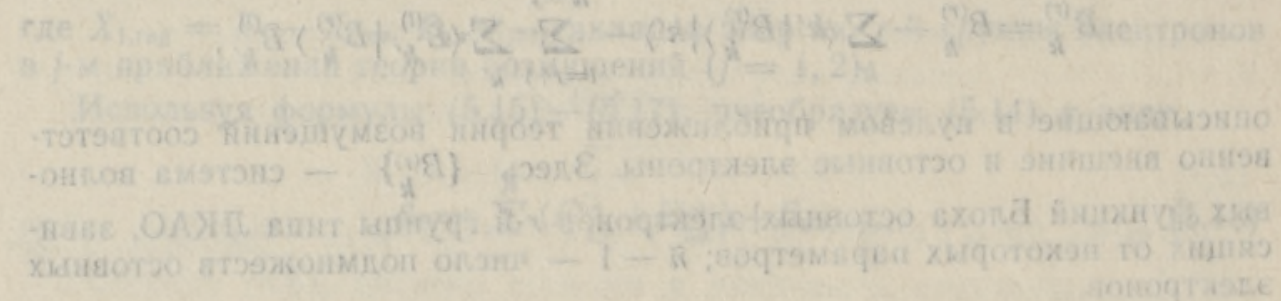

